\title{
Bilingual Statistical Historical Atlas of Israel
}

\author{
Oren Raz
}

Central Bureau of Statistics (Israel), orenr@cbs.gov.il

Keywords: Thematic maps, Atlas, Statistical Abstract

\begin{abstract}
:
State of Israel - 70 Years of Statistics, Statistical Historical Atlas 1948-2018, is an atlas which includes a large collection of statistical data of interest on the State of Israel. These data were collected by the Central Bureau of Statistics over the course of 70 years, starting with the first census in 1948 and the Statistical Abstract of Israel No. 1, continuing with the data of the population censuses over the years, and ending with updated maps from the Statistical Abstract of Israel, which contain data on the State's 70th year of independence.
\end{abstract}

The objectives of the atlas are as follows: 1) Production of a series of maps with historical statistics on the State of Israel, based on data from the Central Bureau of Statistics; 2) Republishing statistical maps with historical value; 3) Comparison of several interesting statistical topics among the various censuses; 4) Providing an up-to-date picture of society in Israel in the 70th year of the establishment of the state through maps.

In order to prepare the maps that show historical data, it was necessary to locate copious information that has appeared in CBS publications over the years, and transfer it to a digital database, using digital scanning of existing maps or through computerized data entry. Thus, much of the information that was stored in old and rare paper publications now finds its way anew to the public and sheds light on the composition and characteristics of Israeli society from the establishment of the State to this day.

Of particular interest in this atlas is the chapter dealing with the Hebrew settlement in 1948, which contains detailed mapping and diagrams of the results of the 1948 census, before the large waves of immigration that arrived after the end of the War of Independence.

The atlas highlights the Population and Housing Census, the flagship of every Central Bureau of Statistics worldwide, and the Statistical Abstract of Israel, which has been published continuously since the establishment of the State. The data of the censuses in this atlas are mapped according to natural regions arranged by the Central Bureau of Statistics and adapted for statistical mapping at the national level. The boundaries of the natural regions are updated for each census and also between the censuses, in accordance with changes in the official administrative divisions of the State of Israel into districts and sub-districts.

The chapters are arranged in chronological order: 1) Population Registration 1948; 2) Population Censuses in Israel; 3) Israel in the 70th Year of its Independence; 4) Selected Topics in a Multi-Year Comparison. The bibliography list is also sorted chronologically by the census years and then by order of publishing.

Some of the maps are based on data typed specifically for this atlas from old CBS publications, and on original historical maps published by the CBS before the digital age. In several cases, historical maps were restored digitally with GIS with up-to-date data. These maps are presented side-by-side, historical map next to its restoration (figure 1). This allows comparisons between the years.

The atlas is bilingual - Hebrew and English. Many of the titles, texts and definitions were translated into English especially for the atlas. The atlas utilizes the different directionality of the two languages in its design- On a two-page spread the right page will receive chapter indications, copyrights and general remarks in Hebrew, and a mirror translation on the left page (figure 2). On the single page level, titles in both languages are first aligned horizontally and then to their matching side. The map titles and legends are also bilingual, with Hebrew on the top in bold letters and item No., and English usually below.

The English introductions, explanatory notes, terms and definitions, bibliography and index were placed in what the Hebrew reader would consider the back of the publication. But to the English reader it would seem natural to read the atlas left to right, so it gives a feeling that the atlas can be read from both sides. However, the chronological order of the chapters dictates flipping the pages from right to left. Texts that are part of the chapters are presented side-by-side - on a single page with a line separator for short texts or on two separate pages for longer texts., English on the left and Hebrew on the right. 


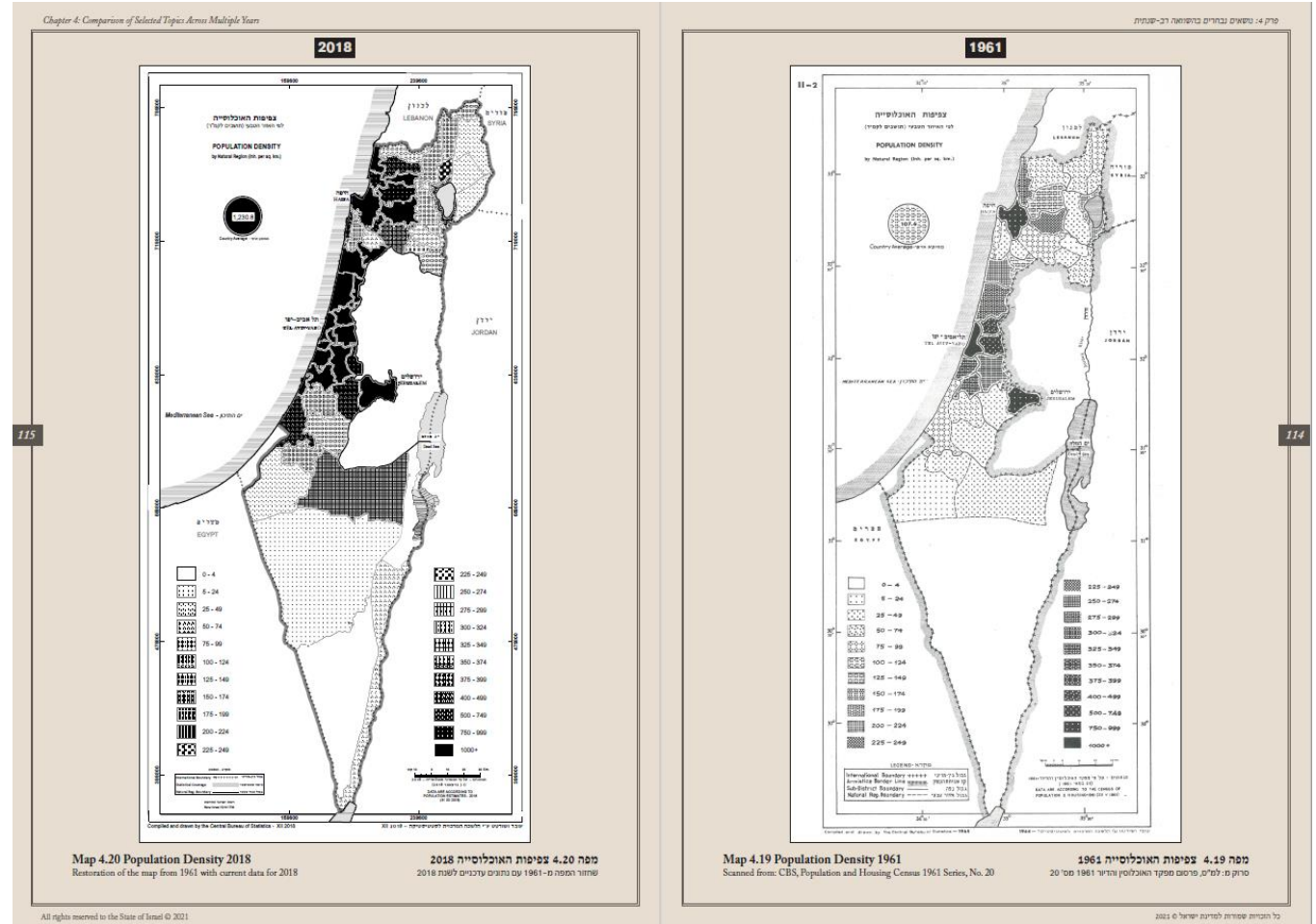

Figure 1. An original historical map of population density (1961) to the right, with its reproduction (2018) to the left.

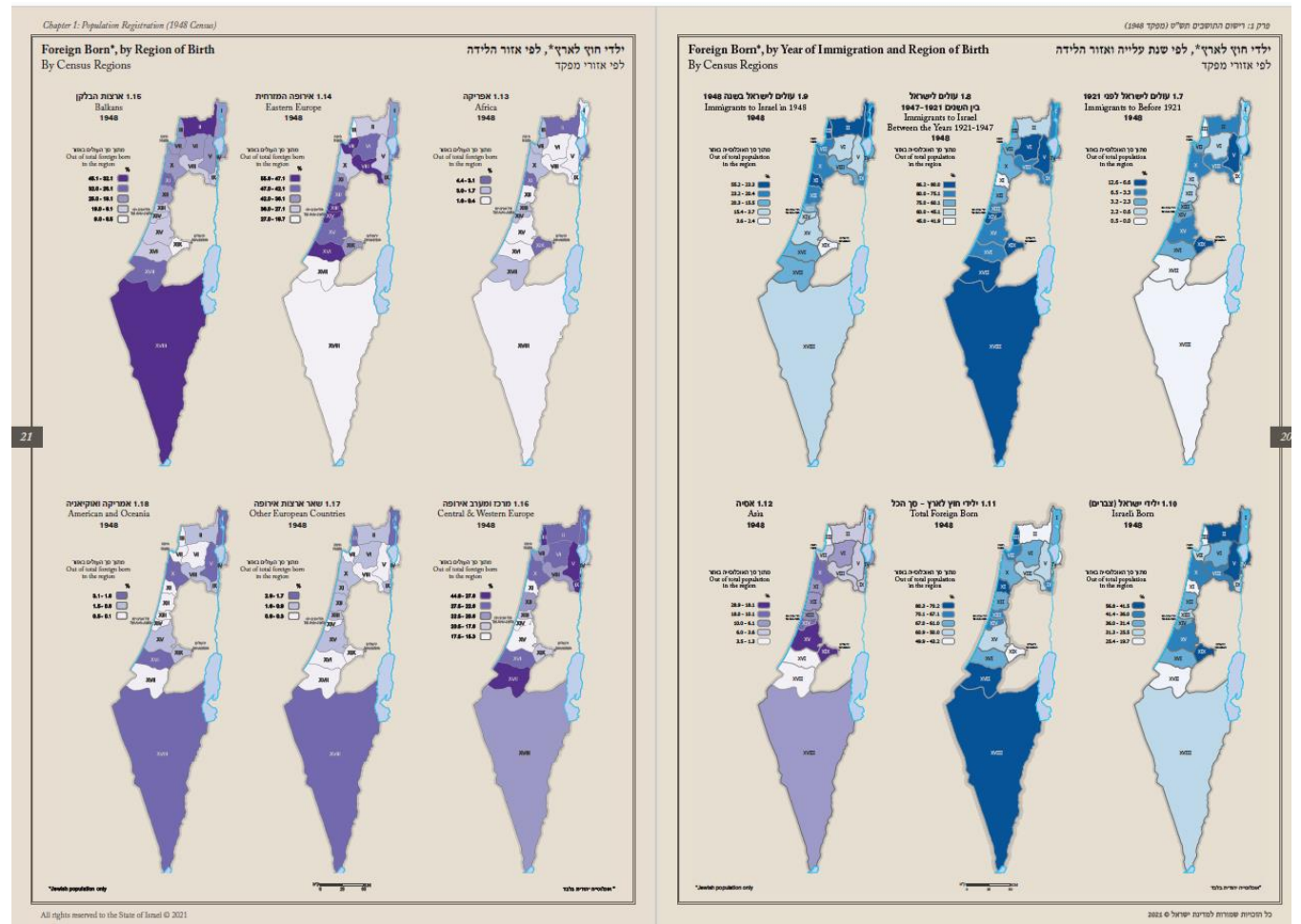

Figure 2. A two-page spread with thematic maps, bilengual by language directionality.

The English terms and definitions, bibliography and index are published separately form the Hebrew, as their alphabetical order differs. However, the chronological order of the chapters dictates flipping the pages from right to left. The table of contents therefore marks the paging in the order they are supposed to be read, with ascending or descending page numbers, respectively to the order of each component type in the atlas - chapters or introductions. The composition of the atlas allows both language readers to enjoy a pleasing cartographic array of historical maps, comparisons of topics and a novel series of statistical thematic maps with a contemporary design. 